\title{
APPROXIMATION AND SPECTRAL PROPERTIES OF PERIODIC SPLINE OPERATORS
}

\author{
by S. L. LEE and W. S. TANG
}

(Received 26th September 1989)

\begin{abstract}
We consider discrete convolution operators $t_{k}^{(\alpha)}$ whose range is the $k$-dimensional space $\mathscr{P}_{k}$ spanned by the translates of a single function. Examples of $\mathscr{S}_{k}$ include the space of trigonometric polynomials, periodic polynomial splines and trigonometric splines. The eigenfunctions of these operators corresponding to the nonzero eigenvalues are independent of $\alpha$, and they form an orthogonal basis for $\mathscr{S}_{k}$. The limiting behaviour of $t_{k}^{(\alpha)}$ as $\alpha, k \rightarrow \infty$, is also considered. The corresponding limiting semigroups are computed explicitly.
\end{abstract}

1980 Mathematics subject classification (1985 Revision): Primary 41A15, 41A10, 42A10, Secondary 47DO5.

\section{Introduction}

For every positive integer $k$, let $\phi_{k}$ be an essentially bounded, measurable, complexvalued $2 \pi$-periodic function defined on $\mathbb{R}$, with Fourier series

$$
\phi_{k}(x) \sim \sum_{\nu} \hat{\phi}_{k, v} e^{i v x}
$$

where

$$
\hat{\phi}_{k, v}=\frac{1}{2 \pi} \int_{-\pi}^{\pi} \phi_{k}(x) e^{-i v x} d x, \quad v \in \mathbb{Z}
$$

Let $X_{2 \pi}$ be the Banach space $C_{2 \pi}$ of all continuous complex-valued $2 \pi$-periodic functions on $\mathbb{R}$, or the space $L_{2 \pi}^{p}$ of all complex-valued $2 \pi$-periodic $L^{p}$-functions on $\mathbb{R}$, $1 \leqq p<\infty$. For $X_{2 \pi}=C_{2 \pi}$, we further assume that $\phi_{k}$ is continuous. Let $h:=2 \pi / k, \omega:=e^{i h}$ and suppose that $\phi_{k}(\cdot-j h), j=0,1, \ldots, k-1$, span a $k$-dimensional subspace $\mathscr{F}_{k}$ of $X_{2 \pi}$.

Define $T_{k}^{(0)}:=I$, the identity operator on $X_{2 \pi}$. For every positive integer $\alpha$, define

$$
\phi_{k}^{(\alpha)}:=\phi_{k} * \cdots * \phi_{k} \quad(\alpha \text { times }),
$$

the convolution of $\phi_{k}$ with itself $\alpha$ times, and for $f \in X_{2 \pi}$, define

$$
\left(T_{k}^{(\alpha)} f\right)(x):=\left(\phi_{k}^{(\alpha)} * f\right)(x)
$$

and 


$$
\left(t_{k}^{(\alpha)} f\right)(x):=\frac{1}{k} \sum_{j=0}^{k-1}\left(T_{k}^{(\alpha)} f\right)(j h) \phi_{k}(x-j h) .
$$

For $f \in C_{2 \pi}, t_{k}^{(0)} f$ is also defined by (1.4).

Examples of $\phi_{k}$ and the corresponding subspace $\mathscr{S}_{k}$ include

(i) de la Vallée Poussin kernel

$$
\phi_{k}(x) \equiv \chi_{m}(x):=\sum_{v=-m}^{m} \frac{(m !)^{2}}{(m-v) !(m+v) !} e^{i v x}
$$

where $k=2 m+1$ (see $[1,3,14]$ and $\mathscr{S}_{k}$ is the space of trigonometric polynomials of degree $m$,

(ii) uniform trigonometric $B$-spline $\tau_{m, k}$ which generates the space of uniform trigonometric splines $\mathscr{T}_{k}([16,17])$ which is studied in Section 5 ,

(iii) periodic polynomial $B$-spline $b_{n, k}$ and $\mathscr{S}_{k}$ is the space of periodic polynomial splines (see $[13,15])$.

Interpolation by linear combinations of translates of $\phi_{k}$ has been studied in [5] and [11]. In this note we shall study the approximation and spectral properties of the operators $t_{k}^{(\alpha)}$ defined by (1.4). The spectral properties of $t_{k}^{(\alpha)}$ are studied in Section 2 where their eigenvalues and eigenvectors are obtained explicitly using the theory of circulant matrices. The eigenfunctions of $t_{k}^{(\alpha)}$ corresponding to nonzero eigenvalues are independent of $\alpha$, and they form an orthonormal basis for $\mathscr{S}_{k}$. In Section 3 we study the limiting behaviour of $t_{k}^{(\alpha)} f$ as $\alpha, k \rightarrow \infty$, which is similar to the iterates of positive convolution operators [9]. The general theories of Sections 2 and 3 are applied to periodic polynomial splines in Section 4 and to trigonometric splines in Section 5 . The resulting orthonormal periodic polynomial splines in Section 4 are the same as those considered recently in [8]. In Section 5 we show that the corresponding set of orthonormal trigonometric splines of degree $m$ contains the finite section $\left\{e^{i v x}:-m \leqq v \leqq\right.$ $m\}$ of the orthonormal Fourier system. In this case, the corresponding operator $t_{m, k}^{(\alpha)} f$, with $\alpha=0$, is a discrete analogue of the convolution operator with trigonometric $B$ spline kernel which was studied in [7].

\section{The spectral properties of $t_{k}^{(\alpha)}$}

For any positive integer $\alpha$, the operators $T_{k}^{(\alpha)}$ and $t_{k}^{(\alpha)}$ defined on $X_{2 \pi}$ by (1.3) and (1.4) can be written as

$$
\left(T_{k}^{(x)} f\right)(x)=\frac{1}{2 \pi} \int_{-\pi}^{\pi} \phi_{k}^{(\alpha)}(x-t) f(t) d t
$$

and 


$$
\left(t_{k}^{(\alpha)} f\right)(x)=\frac{1}{2 \pi} \int_{-\pi}^{\pi} \psi_{k}^{(\alpha)}(x, t) f(t) d t
$$

where

$$
\phi_{k}^{(\alpha)}(x) \sim \sum_{v} \hat{\phi}_{k, v}^{\alpha} e^{i v x}
$$

and

$$
\psi_{k}^{(\alpha)}(x, t):=\frac{1}{k} \sum_{j=0}^{k-1} \phi_{k}^{(\alpha)}(j h-t) \phi_{k}(x-j h)
$$

For $\alpha=0$, the operator $t_{k}^{(0)}$ defined on $C_{2 \pi}$ is given by

$$
\left(t_{k}^{(0)} f\right)(x)=\frac{1}{k} \sum_{j=0}^{k-1} f(j h) \phi_{k}(x-j h) .
$$

These are linear operators on $X_{2 \pi}$, and they are positive if $\phi_{k}$ is positive.

For every nonnegative integer $\alpha$, the matrix of $t_{k}^{(\alpha)}: \mathscr{S}_{k} \rightarrow \mathscr{S}_{k}$ with respect to the basis $\left\{\phi_{k}(\cdot-j h): j=0,1, \ldots, k-1\right\}$ is the $k \times k$ matrix $G^{(\alpha)}:=\left[g_{i, m}^{(\alpha)}\right] / k$, where

$$
g_{l, m}^{(\alpha)}:=\left(T_{k}^{(\alpha)} \phi_{k}(\cdot-m h)\right)(l h)
$$

for $l, m=0,1, \ldots, k-1$. If $\alpha \geqq 1$, by (2.1),

$$
\begin{aligned}
g_{l, m}^{(\alpha)} & =\frac{1}{2 \pi} \int_{-\pi}^{\pi} \phi_{k}^{(\alpha)}(l h-t) \phi_{k}(t-m h) d t \\
& =\phi_{k}^{(\alpha+1)}((l-m) h) .
\end{aligned}
$$

Since $T_{k}^{(0)}=I$, this last expression for $g_{l, m}^{(\alpha)}$ is still valid when $\alpha=0$.

Hence

$$
g_{l, m}^{(\alpha)}=\phi_{k}^{(\alpha+1)}((l-m) h) \text { if } \alpha \geq 0 \text { and } l, m=0,1, \ldots, k-1
$$

It can be shown easily that each $G^{(\alpha)}$ is a circulant matrix. The spectral properties of circulant matrices are well-known (see [4, p. 73]). The eigenvalues of $G^{(\alpha)}$ are

$$
\lambda_{k, j}^{(\alpha)} \equiv \lambda_{j}^{(\alpha)}:=\frac{1}{k} \sum_{m=0}^{k-1} g_{0, m}^{(\alpha)} \omega^{j m}, \quad j=0,1, \ldots, k-1,
$$

and the corresponding eigenvectors are $\left(1, \omega^{j}, \ldots, \omega^{(k-1) j}\right)^{T}, j=0,1, \ldots, k-1$. Hence the eigenvalues of $t_{k}^{(\alpha)}: \mathscr{S}_{k} \rightarrow \mathscr{S}_{k}$ are 


$$
\begin{aligned}
\lambda_{k, j}^{(\alpha)} \equiv \lambda_{j}^{(\alpha)} & =\frac{1}{k} \sum_{m=0}^{k-1} \phi_{k}^{(\alpha+1)}(-m h) \omega^{j m} \\
& =\frac{1}{k} \sum_{l=0}^{k-1} \phi_{k}^{(\alpha+1)}(l h) \omega^{-j l}, \quad j=0,1, \ldots, k-1,
\end{aligned}
$$

with corresponding eigenfunctions

$$
\begin{aligned}
f_{k, j} \equiv f_{j}: & =\frac{1}{k} \sum_{l=0}^{k-1} \omega^{j l} \phi_{k}(\cdot-l h) \\
& =\frac{1}{k} \sum_{l=0}^{k-1} \omega^{-j l} \phi_{k}(\cdot+l h), \quad j=0,1, \ldots, k-1,
\end{aligned}
$$

which are independent of $\alpha$.

For $f, g \in L_{2 \pi}^{2}$, let

$$
\langle f, g\rangle:=\frac{1}{2 \pi} \int_{-\pi}^{\pi} f(t) \overline{g(t)} d t
$$

be the inner product of $f$ and $g$. We summarise some properties of $\lambda_{j}^{(\alpha)}$ and $f_{j}$ in the following:

Theorem 2.1. For $j=0,1, \ldots, k-1$,

$$
\begin{gathered}
f_{j}(\cdot+h)=\omega^{j} f_{j}, \\
f_{j}(x) \sim \sum_{p \in \mathbf{Z}} \hat{\phi}_{k, j+k p} e^{i x(j+k p)}, \\
\left\langle f_{j}, f_{l}\right\rangle=0 \quad \text { if } j \neq l, \\
\left\|f_{j}\right\|_{2}=\left(\sum_{p \in \mathbb{Z}}\left|\hat{\phi}_{k, j+k p}\right|^{2}\right)^{1 / 2}, \\
\lambda_{j}^{(0)}=f_{j}(0), \\
\lambda_{j}^{(\alpha)}=\sum_{p \in \mathbf{Z}} \hat{\phi}_{k, j+k p}^{\alpha+1} \quad \text { for } \quad \alpha \geqq 1 .
\end{gathered}
$$

Moreover, 
(i) if $\phi_{k}$ admits the Fourier expansion

$$
\phi_{k}(x)=\sum_{v \in \mathbb{Z}} \hat{\phi}_{k, v} e^{i v x}, \quad x \in \mathbb{R}
$$

then we have

$$
\begin{aligned}
f_{j}(x) & =\sum_{p \in \mathbb{Z}} \hat{\phi}_{k, j+k_{p}} e^{i x(j+k p)}, \quad x \in \mathbb{R}, \quad \text { and } \\
\lambda_{j}^{(0)} & =\sum_{p \in \mathbf{Z}} \hat{\phi}_{k, j+k_{p}} \text { for } j=0,1, \ldots, k-1 ;
\end{aligned}
$$

(ii) if $\phi_{k}$ is real-valued, then $f_{0}$ and $\lambda_{0}^{(\alpha)}$ are real-valued, $f_{j}=\bar{f}_{k-j}$ and $\lambda_{j}^{(\alpha)}=\overline{\lambda_{k-j}^{(\alpha)}}$ for $\alpha \geqq 0$ and $1 \leqq j \leqq k-1$;

(iii) if $\phi_{k}$ is real-valued and even, then

$$
f_{j}(x)=\overline{f_{j}(-x)}, \quad \lambda_{j}^{(\alpha)}=\left\langle f_{j}, \phi_{k}^{(\alpha)}\right\rangle
$$

for $0 \leqq j \leqq k-1$ and $\alpha \geqq 1$, and

$$
\lambda_{j}^{(\alpha)}=\lambda_{k-j}^{(\alpha)}
$$

for $1 \leqq j \leqq k-1$ and $\alpha \geqq 0$.

Proof. The relation (2.9) follows from (2.8) and a change of variable. By (2.8) again, the Fourier coefficients of $f_{j}$ are

$$
\begin{aligned}
\hat{f}_{j, v} & =\frac{1}{k} \sum_{l=0}^{k-1} \omega^{j l} \hat{\phi}_{k, v} e^{-i v l h} \\
& =\hat{\phi}_{k, v}\left(\frac{1}{k} \sum_{l=0}^{k-1} \omega^{(j-v) l}\right), \quad v \in \mathbb{Z},
\end{aligned}
$$

which is 0 if $v \not \equiv j(\bmod k)$, and is $\hat{\phi}_{k, j+k p}$ if $v=j+k p$ for some $p \in \mathbb{Z}$. Hence (2.10) holds, and from which (2.11) and (2.12) follow. Comparing (2.7) and (2.8), we obtain (2.13). Since $\phi_{k}$ is essentially bounded, $\phi_{k}^{(\alpha+1)}=\phi_{k}^{(\alpha)} * \phi_{k}$ is continuous with its Fourier transform in $l^{1}$ for $\alpha \geqq 1$. Hence

$$
\phi_{k}^{(a+1)}(x)=\sum_{v \in Z} \hat{\phi}_{k, v}^{\alpha+1} e^{i v x}
$$

where the Fourier series on the right hand side converges absolutely for every $x$ in $\mathbb{R}$. By (2.7) and (2.17), for $\alpha \geqq 1$,

$$
\lambda_{j}^{(\alpha)}=\frac{1}{k} \sum_{l=0}^{k-1}\left(\sum_{v \in \mathbf{Z}} \hat{\phi}_{k, v}^{\alpha+1} \omega^{v l}\right) \omega^{-j l}
$$




$$
\begin{aligned}
& =\sum_{v \in Z} \hat{\phi}_{k, v}^{\alpha+1}\left(\frac{1}{k} \sum_{l=0}^{k-1} \omega^{(v-j) l}\right) \\
& =\sum_{p \in Z} \hat{\phi}_{k, j+k p}^{\alpha+1}, \quad j=0,1, \ldots, k-1 .
\end{aligned}
$$

Hence (2.14) holds. The rest of the assertions in the theorem are easy consequences of (2.7) and (2.8) and their proofs are omitted.

Corollary 2.2. For $j=0,1, \ldots, k-1$, let

$$
E_{j}:=f_{j} /\left\|f_{j}\right\|_{2}
$$

Then $\left\{E_{j}: j=0,1, \ldots, k-1\right\}$ is an orthonormal basis of $\mathscr{S}_{k}$ consisting of eigenfunctions of $t_{k}^{(\alpha)}$.

Proposition 2.3. Suppose that for some $\alpha \geqq 0$,

$$
\lambda_{l}^{(a)} \neq 0, \quad l=0,1, \ldots, k-1
$$

If $e_{j}(x):=e^{i j x} \in \mathscr{S}_{k}$ for some $j \in\{0,1, \ldots, k-1\}$, then $\hat{\phi}_{k, j+k p}=0$ for every $p \in \mathbb{Z} \backslash\{0\}$, $f_{j}=\hat{\phi}_{k, j} e_{j}, E_{j}=\hat{\phi}_{k, j} e_{j} /\left|\hat{\phi}_{k, j}\right|, \lambda_{j}^{(\beta)}=\widehat{\phi}_{k, j}^{\beta+1}$ and

$$
t_{k}^{(\beta)} e_{j}=\hat{\phi}_{k, j}^{\beta+1} e_{j}
$$

for every integer $\beta \geqq 0$.

Proof. By the definition of $T_{k}^{(0)}$ and (2.1), $T_{k}^{(\beta)} e_{j}=\hat{\phi}_{k, j}^{\beta} e_{j}$ for every integer $\beta \geqq 0$ (where $\hat{\phi}_{k, j}^{0}=1$ ). Hence by (1.4),

$$
\begin{aligned}
t_{k}^{(\beta)} e_{j} & =\frac{1}{k} \sum_{l=0}^{k-1} \hat{\phi}_{k, j}^{\beta} e_{j}(l h) \phi_{k}(\cdot-l h) \\
& =\hat{\phi}_{k, j}^{\beta} f_{j} .
\end{aligned}
$$

On the other hand, $t_{k}^{(\beta)} f_{j}=\lambda_{j}^{(\beta)} f_{j}$. Since $t_{k}^{(\alpha)}: \mathscr{S}_{k} \rightarrow \mathscr{T}_{k}$ is injective by (2.19),

$$
f_{j}=\lambda_{j}^{(\alpha)} \hat{\phi}_{k, j}^{-a} e_{j}
$$

By (2.10) and (2.21), $\hat{\phi}_{k, j+k p}=0$ for $p \in \mathbb{Z} \backslash\{0\}, \lambda_{j}^{(a)}=\hat{\phi}_{k, j}^{\alpha+1}$ and $f_{j}=\hat{\phi}_{k, j} e_{j}$. Hence (2.20) holds and $\lambda_{j}^{(\beta)}=\hat{\phi}_{k, j}^{\beta+1}$ for every $\beta \geqq 0$ by (2.13) and (2.14). Finally,

$$
E_{j}=f_{j} /\left\|f_{j}\right\|_{2}=\hat{\phi}_{k, j} e_{j} /\left|\hat{\phi}_{k, j}\right| \text {. }
$$




\section{Approximation properties of $t_{k}^{(\alpha)}$}

Throughout this section, suppose that each $\phi_{k}$ is continuous, positive, $2 \pi$-periodic with Fourier expansion

$$
\phi_{k}(x)=\sum_{v \in \mathbf{Z}} \hat{\phi}_{k, v} e^{i v x}, \quad x \in \mathbb{R}
$$

such that

$$
\begin{gathered}
\hat{\phi}_{k, 0}=1, \\
\lim _{k \rightarrow \infty} \hat{\phi}_{k, 1}=1, \\
\hat{\phi}_{k, k p}=0 \text { for every } p \in \mathbb{Z} \backslash\{0\}, \\
\lim _{k \rightarrow \infty} \hat{\phi}_{k, 1+k p}=0 \text { for every } p \in \mathbb{Z} \backslash\{0\}, \text { and }
\end{gathered}
$$

there exist a positive integer $K$ and an absolutely convergent series $\sum_{p \neq 0} b_{p}$ such that

$$
\left|\hat{\phi}_{k, 1+k p}\right| \leqq\left|b_{p}\right| \text { if } k \geqq K \text { and } p \neq 0 \text {. }
$$

It follows from the positivity of $\phi_{k},(3.2),(3.3)$ and Korovkin's Theorem (see [2, Proposition 1.3.10]) that

$$
\lim _{k \rightarrow \infty} \hat{\phi}_{k, j}=1 \quad \text { for every } j \in \mathbb{Z}
$$

Lemma 3.1. Let $k$ and $\alpha$ be positive integers, $h=2 \pi / k$, and $\phi_{k}^{(\alpha)}$ and $\psi_{k}^{(\alpha)}$ be defined by (1.2) and (2.3) respectively. Then

$$
\begin{gathered}
\frac{1}{2 \pi} \int_{-\pi}^{\pi} \phi_{k}^{(\alpha)}(t) d t=1, \\
\frac{1}{k} \sum_{l=0}^{k-1} \phi_{k}^{(\alpha)}(\cdot-l h)=\frac{1}{k} \sum_{t=0}^{k-1} \phi_{k}^{(\alpha)}(\cdot+l h)=1, \text { and } \\
\frac{1}{2 \pi} \int_{-\pi}^{\pi} \psi_{k}^{(\alpha)}(\cdot, t) d t=\frac{1}{2 \pi} \int_{-\pi}^{\pi} \psi_{k}^{(\alpha)}(t, \cdot) d t=1,
\end{gathered}
$$

Proof. The relation (3.8) follows directly from (3.2). The first equality in (3.9) follows by a change of variable. By (3.1), for every $x \in \mathbb{R}$

$$
\phi_{k}^{(\alpha)}(x)=\sum_{v \in \mathbf{Z}} \hat{\phi}_{k, v}^{\alpha} e^{i v x}
$$


Hence

$$
\begin{aligned}
\frac{1}{k} \sum_{l=0}^{k-1} \phi_{k}^{(\alpha)}(x+l h) & =\sum_{v \in \mathbf{Z}} \hat{\phi}_{k, v}^{\alpha} e^{i v x}\left(\frac{1}{k} \sum_{l=0}^{k-1} e^{i v l h}\right) \\
& =\sum_{p \in Z} \hat{\phi}_{k, k p}^{\alpha} e^{i k p x} \\
& =1
\end{aligned}
$$

by (3.2) and (3.4). Finally, (3.10) follows from (2.3), (3.8) and (3.9).

As a result of Lemma 3.1, for every integer $\alpha \geqq 0$,

$$
T_{k}^{(\alpha)} 1=1 \quad \text { and } \quad t_{k}^{(\alpha)} 1=1
$$

Proposition 3.2. For $\alpha \geqq 0$ and $f \in X_{2 \pi}\left(f \in C_{2 \pi}\right.$ if $\left.\alpha=0\right)$,

$$
\left\|t_{k}^{(\alpha)} f\right\|_{x_{2 \pi}} \leqq\|f\|_{x_{2 \pi}}
$$

For $\alpha \geqq 1$ and $f \in L_{2 \pi}^{1}$,

$$
\int_{-\pi}^{\pi}\left(t_{k}^{(\alpha)} f\right)(x) d x=\int_{-\pi}^{\pi} f(x) d x
$$

Proof. The relation (3.12) for $X_{2 \pi}=C_{2 \pi}$ follows from (2.2) and (3.10) for the case $\alpha>0$, and from (2.4) and (3.9) for $\alpha=0$. For $X_{2 \pi}=L_{2 \pi}^{p}, 1 \leqq p<\infty$, let $1 / p+1 / q=1$. By (2.2), Hölder's inequality and (3.10),

$$
\begin{aligned}
\left|\left(t_{k}^{(\alpha)} f\right)(x)\right| & \leqq\left(\frac{1}{2 \pi} \int_{-\pi}^{\pi} \psi_{k}^{(\alpha)}(x, t) d t\right)^{1 / q}\left(\frac{1}{2 \pi} \int_{-\pi}^{\pi} \psi_{k}^{(\alpha)}(x, t)|f(t)|^{p} d t\right)^{1 / p} \\
& =\left(\frac{1}{2 \pi} \int_{-\pi}^{\pi} \psi_{k}^{(\alpha)}(x, t)|f(t)|^{p} d t\right)^{1 / p}
\end{aligned}
$$

Hence by (3.10),

$$
\begin{aligned}
\left\|t_{k}^{(\alpha)} f\right\|_{p}^{p} & \leqq \frac{1}{2 \pi} \int_{-\pi}^{\pi}\left(\frac{1}{2 \pi} \int_{-\pi}^{\pi} \psi_{k}^{(\alpha)}(x, t) d x\right)|f(t)|^{p} d t \\
& =\|f\|_{p}^{p} .
\end{aligned}
$$


The relation (3.13) also follows from (3.10) and (2.2).

Proposition 3.3. For every $f \in C_{2 \pi}$,

$$
\left(t_{k}^{(0)} f\right)(x)=\frac{1}{k} \sum_{i=0}^{k-1} f(l h) \phi_{k}(x-l h) \rightarrow f(x)
$$

uniformly on $\mathbb{R}$ as $k \rightarrow \infty$.

Proof. We first prove that (3.14) holds for $f=e_{1}$, where $e_{1}(x)=e^{i x}$. By (3.6), Lebesgue's Dominated Convergence Theorem and (3.5), we have

$$
\lim _{k \rightarrow \infty} \sum_{p \neq 0}\left|\hat{\phi}_{k, 1+k p}\right|=\sum_{p \neq 0} \lim _{k \rightarrow \infty}\left|\hat{\phi}_{k, 1+k p}\right|=0 \text {. }
$$

Hence for every $x \in \mathbb{R}$,

$$
\begin{aligned}
\left|\left(t_{k}^{(0)} e_{1}\right)(x)-e_{1}(x)\right| & =\left|e^{i x}\left(\sum_{p \in \mathbf{Z}} \hat{\phi}_{k, 1+k p} e^{i k p x}-1\right)\right| \\
& \leqq\left|\hat{\phi}_{k, 1}-1\right|+\sum_{p \neq 0}\left|\hat{\phi}_{k, 1+k p}\right|
\end{aligned}
$$

which tends to 0 as $k \rightarrow \infty$ by (3.3) and (3.15). Thus

$$
\lim _{k \rightarrow \infty}\left\|t_{k}^{(0)} e_{1}-e_{1}\right\|_{c_{2 \pi}}=0
$$

It follows from this relation, the positivity of the operators $t_{k}^{(0)},(3.11)$ and Korovkin's Theorem that (3.14) holds for every $f \in C_{2 \pi}$.

Remarks. 1. Suppose that each $\phi_{k}$ is continuous, positive, $2 \pi$-periodic satisfying (3.1), (3.2), (3.4) and

$$
\lim _{k \rightarrow \infty} \sup _{\delta \leqq|x| \leqq \pi}\left|\phi_{k}(x)\right|=0 \quad \text { for every } 0<\delta<\pi
$$

Then (3.7), Lemma 3.1, Proposition 3.2 and Proposition 3.3 are still valid.

2. For $1 \leqq p<\infty$, by Proposition 3.3, (3.12) and density of trigonometric polynomials in $L_{2 \pi}^{p}$,

$$
\lim _{k \rightarrow \infty}\left\|t_{k}^{(0)} f-f\right\|_{L_{2 \pi}^{p}}=0 \quad \text { for every } f \in L_{2 \pi}^{p}
$$


Theorem 3.4. Let $\left\{\alpha_{k}\right\}_{k \geqq 1}$ be a nondecreasing sequence of positive integers. $A$ necessary and sufficient condition for $\left\{t_{k}^{\left(\alpha_{k}\right)} f\right\}_{k \geqq 1}$ to converge strongly in $X_{2 \pi}$ as $k \rightarrow \infty$ for every $f \in X_{2 \pi}$ is that

$$
\lim _{k \rightarrow \infty} \hat{\phi}_{k, v}^{\alpha_{k}} \text { exists for all } v \in \mathbb{Z}
$$

Furthermore,

$$
\lim _{k \rightarrow \infty}\left\|t_{k}^{\left(\alpha_{k}\right)} f-f\right\|_{x_{2 \pi}}=0 \quad \text { for all } f \in X_{2 \pi}
$$

if and only if

$$
\lim _{k \rightarrow \infty} \hat{\phi}_{k, 1}^{\alpha_{k}}=1
$$

and

$$
\lim _{k \rightarrow \infty}\left\|t_{k}^{\left(\alpha_{k}\right)} f-\hat{f}_{0}\right\|_{x_{2 \pi}}=0 \quad \text { for all } f \in X_{2 \pi}
$$

if and only if

$$
\lim _{k \rightarrow \infty} \hat{\phi}_{k, v}^{\alpha_{k}}=0 \quad \text { for all } v \in \mathbb{Z} \backslash\{0\}
$$

Proof. If $e_{v}(x)=e^{i v x}, v \in \mathbb{Z}$, then

$$
\left(t_{k}^{\left(\alpha_{k}\right)} e_{v}\right)(x)=\hat{\phi}_{k, v}^{\alpha_{k}}\left(\frac{1}{k} \sum_{j=0}^{k-1} e_{v}(j h) \phi_{k}(x-j h)\right)
$$

by (2.1) and (1.4). Proposition 3.3 and (3.19) imply that $t_{k}^{\left(z_{k}\right)} e_{v}$ converges strongly in $X_{2 \pi}$ as $k \rightarrow \infty$ for all $v \in \mathbb{Z}$ if and only if (3.16) holds. Since $\left\{t_{k}^{\left(\alpha_{k}\right)}\right\}_{k \geqq 1}$ is uniformly bounded, the first part of Theorem 3.4 follows from the Banach-Steinhaus Theorem.

The relation (3.17) follows from Korovkin's Theorem, since (3.19) with $v=1$, and Proposition 3.3 imply that $t_{k}^{\left(\alpha_{k}\right)} e_{1} \rightarrow e_{1}$ strongly in $X_{2 \pi}$ as $k \rightarrow \infty$ if and only if $\lim _{k \rightarrow \infty} \hat{\phi}_{k, 1}^{\alpha_{k}}=1$.

By (3.11) and (3.19), $t_{k}^{\left(\alpha_{k}\right)} e_{v} \rightarrow \delta_{0, v}$ in $X_{2 \pi}$ as $k \rightarrow \infty$ if and only if $\lim _{k \rightarrow \infty} \hat{\phi}_{k, v}^{\alpha_{k}}=0, v \neq 0$. Hence (3.18) holds.

The results (3.17) and (3.18) correspond to two special cases of the limit (3.16). We now consider the general situation. Because of $(3.2),\left|\hat{\phi}_{k, v}\right| \leqq 1$ for all $v \in \mathbb{Z}$. Let

$$
\hat{\phi}_{k, v}:=1-\varepsilon_{k, v}, \quad v \in \mathbb{Z}
$$


By (3.7), $\lim _{k \rightarrow \infty} \varepsilon_{k, v}=0$ for all $v \in \mathbb{Z}$. The limit (3.16) exists if

$$
\lim _{k \rightarrow \infty} \alpha_{k} \varepsilon_{k, v}:=\xi_{v} \text { exists, }
$$

where

$$
\operatorname{Re} \xi_{v} \in \mathbb{R} \cup\{+\infty\} \text { and } \operatorname{Im} \xi_{v} \in \mathbb{R}, \quad v \in \mathbb{Z}
$$

In this case,

$$
\lim _{k \rightarrow \infty} \hat{\phi}_{k, v}^{\alpha_{k}}=e^{-\xi_{v}}, \quad v \in \mathbb{Z}
$$

Theorem 3.5. Let $\left\{\alpha_{k}\right\}_{k \geqq 1}$ be a nondecreasing sequence of positive integers. If (3.20) holds, then $\left\{t_{k}^{\left(\alpha_{k}\right)} f\right\}$ converges strongly in $X_{2 \pi}$ for every $f \in X_{2 \pi}$. In this case, for any $\zeta>0$ and $f \in X_{2 \pi}$,

$$
\lim _{k \rightarrow \infty}\left\|t_{k}^{\left(\left[\alpha_{k} \zeta\right]\right)} f-\Phi_{\zeta} f\right\|_{x_{2 \pi}}=0
$$

where $[x]$ is the greatest integer less than or equal to $x$, and for $f(x) \sim \sum_{v \in \mathrm{Z}} \hat{f}_{v} e^{i v x}$,

$$
\Phi_{\zeta} f(x): \sim \sum_{v \in \mathbf{Z}} e^{-\zeta \xi_{v}} \hat{f}_{v} e^{i v x} .
$$

The operators $\Phi_{\zeta}, \zeta>0$, form a semigroup whose infinitesimal generator $A_{\zeta}$ is characterised by

$$
A_{\zeta} f(x) \sim \sum_{v \in \mathbf{Z}}\left(-\xi_{v} \hat{f}_{v}\right) e^{i v x}
$$

for every $f$ in the domain of $A_{\zeta}$.

Proof. The first part follows from Theorem 3.4 and the above remark. Suppose (3.20) holds and $\zeta>0$. Since $\lim _{k \rightarrow \infty} \hat{\phi}_{k, v}=1$ for all $v \in \mathbb{Z}$ and $\alpha_{k} \zeta-1<\left[\alpha_{k} \zeta\right] \leqq \alpha_{k} \zeta$, by writing $\hat{\phi}_{k, v}=\gamma_{k, v} e^{i \theta_{k, v}}$, where $\gamma_{k, v} \geqq 0$ and $-\pi<\theta_{k, v} \leqq \pi$, it is straightforward that

$$
\lim _{k \rightarrow \infty} \gamma_{k, v}^{\left[\alpha_{k} \zeta\right]}=\lim _{k \rightarrow \infty} \gamma_{k, v}^{\alpha_{k} \zeta}, \quad \lim _{k \rightarrow \infty} e^{\left.i \theta_{k, v} \downarrow \alpha_{k} \zeta\right]}=\lim _{k \rightarrow \infty} e^{i \theta_{k, v} \alpha_{k} \zeta}
$$

and so

$$
\lim _{k \rightarrow \infty} \hat{\phi}_{k, v}^{\left[\alpha_{k}[\zeta]\right.}=\lim _{k \rightarrow \infty} \hat{\phi}_{k, v}^{\alpha_{k} \zeta}=e^{-\zeta \xi_{v}}
$$

By (3.14), (3.19) and (3.25), if $e_{v}(x)=e^{i v x}, v \in \mathbb{Z}$, then

$$
\left(t_{k}^{\left(\left[\alpha_{k} \zeta\right]\right)} e_{v}\right)(x) \rightarrow e^{-\zeta \xi_{v}} e^{i v x}
$$


strongly in $X_{2 \pi}$ as $k \rightarrow \infty$. The results (3.22) and (3.23) follow from the Banach-Steinhaus Theorem. Relation (3.16) for the infinitesimal generator $A_{\zeta}$ of $\Phi_{\zeta}$ follows from (3.23) (see [2]).

Remarks. 1. For a sequence $\left\{c_{k}\right\}$ of complex numbers converging to 1 , the existence of $\lim _{k \rightarrow \infty} c_{k}^{k}$ does not imply that $\lim _{k \rightarrow \infty} k\left(1-c_{k}\right)=\xi$ exists, where $\operatorname{Re} \xi \in \mathbb{R} \cup\{+\infty\}$ and Im $\xi \in \mathbb{R}$. Thus for complex $\hat{\phi}_{k, v}$, conditions (3.16) and (3.20) are not equivalent.

2. If $\hat{\phi}_{k, v}$ are all real (or if all $\phi_{k}$ are positive and even), then (3.16) and (3.20) are equivalent. In this case, (3.20) is a necessary and sufficient condition for $\left\{t_{k}^{\left(\alpha_{k}\right)} f\right\}$ to converge strongly in $X_{2 \pi}$ for every $f \in X_{2 \pi}$.

\section{Periodic polynomial splines}

Let $M_{0}=\chi_{(-1 / 2,1 / 2]}$ and for $n=1,2, \ldots$, let $M_{n}:=M_{0} * M_{n-1}$ be the uniform $B$-spline of degree $n$. Let $k$ be a positive integer, $h:=2 \pi / k$ and for $n=1,2, \ldots$, define

$$
b_{n, k}(x):=\sum_{\nu} k M_{n-1}\left(h^{-1}(x-2 \pi v)\right), \quad x \in \mathbb{R},
$$

the uniform, $2 \pi$-periodic $B$-spline of degree $n-1$. Using the Fourier transform of $M_{n-1}$, a straightforward computation gives

$$
b_{n, k}(x):=\sum_{v} \hat{b}_{n, k, v} e^{i v x}
$$

where

$$
\hat{b}_{n, k, v}:=\left(\frac{\sin h v / 2}{h v / 2}\right)^{n}, \quad v \in \mathbb{Z}
$$

The function $b_{n, k}$ is an even, positive, $2 \pi$-periodic function with $\hat{b}_{n, k, 0}=1, \hat{b}_{n, k, 1} \rightarrow 1$ as $k \rightarrow \infty$ (i.e. $h \rightarrow 0$ ), and it translates $b_{n, k}(x-j h), j=0,1, \ldots, k-1$, span the $k$-dimensional space $\tilde{\mathscr{S}}_{n, k}$ of $2 \pi$-periodic polynomial splines of degree $n-1$ with knots at $j h$ or $\left(j+\frac{1}{2}\right) h$, $j=0,1, \ldots, k-1$, depending on whether $n$ is even or odd (see [15]).

Proposition 4.1. For $\alpha=1,2, \ldots$,

$$
\sum_{p} \hat{b}_{n, k, j+k p}^{\alpha+1} \neq 0, \quad j=0,1, \ldots, k-1 .
$$

Proof. If $j=0$,

$$
\sum_{p} \hat{b}_{n, k, k p}^{\alpha+1}=\hat{b}_{n, k, 0}^{\alpha+1}=1
$$

Suppose $j=1,2, \ldots, k-1$. Then 


$$
\sum_{p} b_{n, k, j+k p}^{\alpha+1}=\left(\sin \frac{h j}{2}\right)^{n(\alpha+1)} \sum_{p}(-1)^{n p(\alpha+1)}\left(\frac{2}{(j+k p) h}\right)^{n(\alpha+1)}
$$

The sum on the right of (4.5) can be expressed as

$$
\begin{aligned}
& \left(\frac{2}{j h}\right)^{n(\alpha+1)}\left\{1+\sum_{p=1}^{\infty}(-1)^{n p(\alpha+1)}\left(\frac{j}{j+k p}\right)^{n(\alpha+1)}\right\} \\
& +\left(\frac{2}{(k-j) h}\right)^{n(\alpha+1)}\left\{1+\sum_{p=2}^{\infty}(-1)^{n p(\alpha+1)}\left(\frac{k-j}{j-k p}\right)^{n(\alpha+1)}\right\} \neq 0 .
\end{aligned}
$$

Hence (4.4) follows from (4.5).

Theorem 2.1 and Proposition 4.1 show that for $\alpha=1,2, \ldots$, the operator

$$
\left(s_{n, k}^{(\alpha)} f\right)(x):=\frac{1}{k} \sum_{j=0}^{k-1}\left(S_{n, k}^{(\alpha)} f\right)(j h) b_{n, k}(x-j h),
$$

where $S_{n, k}^{(\alpha)} f$ is defined by (1.3) with $S_{n, k}^{(\alpha)}:=T_{k}^{(\alpha)}$ and $b_{n, k}=\phi_{k}$, is such that $\left.s_{m, k}^{(\alpha)}\right|_{\tilde{F}_{n, k} \rightarrow \tilde{\mathscr{Y}}_{n, k}}$ is bijective. Hence by (2.14) and (2.8) its nonzero eigenvalues are

$$
\lambda_{n, k, j}^{(\alpha)} \equiv \lambda_{n, j}^{(\alpha)}=\left(\frac{\sin j h / 2}{h / 2}\right)^{n(\alpha+1)} \sum_{p}(-1)^{n p(\alpha+1)} /(j+k p)^{n(\alpha+1)},
$$

with corresponding eigenvectors

$$
f_{n, k, j} \equiv f_{n, j}=\frac{1}{k} \sum_{l=0}^{k-1} \omega^{j l} b_{n, k}(\cdot-l h)
$$

$j=0,1, \ldots, k-1$. It follows from (2.11) and (2.12) in Theorem 2.1 that the orthogonal relations

$$
\left\{\begin{array}{l}
\left\langle f_{n, j}, f_{n, l}\right\rangle=0 \\
\left\|f_{n, j}\right\|_{2}=\sqrt{\lambda_{n, j}^{(1)}}
\end{array} \text { if } \quad j \neq l,\right. \text { and }
$$

hold. This was also established recently in [8]. The normalised eigenfunctions

$$
E_{n, k, j}(x) \equiv E_{n, j}=\frac{1}{\sqrt{\lambda_{n, j}^{(1)}}} f_{n, j}, \quad j=0,1, \ldots, k-1
$$


furnish an orthonormal basis for the space $\tilde{\mathscr{S}}_{n, k}$. Furthermore by $(2.15)$ of Theorem 2.1 , we can write

$$
E_{n, j}(x)=\frac{\sum_{p}(-1)^{n p} e^{i x(j+k p)} /(j+k p)^{n}}{\left(\sum_{p} /(j+k p)^{2 n}\right)^{1 / 2}}, \quad j=0,1, \ldots, k-1 .
$$

Remarks. 1. It was also proved in [8] that if $k$ is odd

$$
E_{n, j}(x) \rightarrow \begin{cases}e^{i j x} & 0 \leqq j<k / 2 \\ e^{i(j-k) x} & k / 2<j \leqq k-1\end{cases}
$$

as $n \rightarrow \infty$. This result follows immediately from (4.11). In fact (4.12) also holds if $k$ is even, and furthermore for $j=k / 2$,

$$
E_{n, k / 2}(x) \rightarrow \cos \frac{k x}{2} \quad \text { as } n \rightarrow \infty
$$

2. Since $\hat{b}_{n, k, v}$ satisfies (3.4), (3.5) and (3.6), the results of Section 3 hold for the operators $s_{n, k}^{(\alpha)}$.

The operators $s_{n, k}^{(a)}$ contain an additional parameter $n$ which plays much the same role as $\alpha$. We shall state, without proof, results on the limiting behaviour of $s_{n, k}^{\left(\alpha_{k}\right)}$ as $n$ and $k$ tend to infinity.

Theorem 4.2. (a) Let $\alpha_{k}, k=1,2, \ldots$, be a nondecreasing sequence of positive integers. Then

$$
\lim _{n, k \rightarrow \infty}\left\|s_{n, k}^{\left(\alpha_{k}\right)} f-f\right\|_{X_{2 \pi}}=0 \quad \text { for all } f \in X_{2 \pi}
$$

if and only if

$$
\begin{gathered}
\lim _{n, k \rightarrow \infty}\left(\frac{\sin \pi / k}{\pi / k}\right)^{n \alpha_{k}}=1 \\
\lim _{n, k \rightarrow \infty}\left\|s_{n, k}^{(\alpha)} f-\hat{f}_{0}\right\|_{X_{2 x}}=0 \quad \text { for all } f \in X_{2 \pi}
\end{gathered}
$$

if and only if

$$
\lim _{n, k \rightarrow \infty}\left(\frac{\sin \pi v / k}{\pi v / k}\right)^{n \alpha_{k}}=0 \text { for all } v \neq 0
$$


(b) A necessary and sufficient condition for $\left(s_{n, k}^{\left(\alpha_{k}\right)} f\right)$ to converge strongly for any $f \in X_{2 \pi}$ as $n, k \rightarrow \infty$ is that

$$
\lim _{n, k \rightarrow \infty}\left(\frac{\sin \pi v / k}{\pi v / k}\right)^{n \alpha_{k}} \quad \text { exists for all } v \in \mathbb{Z}
$$

Let

$$
\frac{\sin \pi v / k}{\pi v / k}=1-\varepsilon_{k, v}
$$

where

$$
\varepsilon_{k, v}=\frac{1}{3 !}\left(\frac{\pi v}{k}\right)^{2}+0\left(\frac{1}{k^{4}}\right)
$$

Then (4.16) holds if and only if $\lim _{n, k \rightarrow \infty} n \alpha_{k} / k^{2}=\gamma$ exists or equals $\infty$. Furthermore if (4.16) holds, then

$$
\lim _{n, k \rightarrow \infty}\left(\frac{\sin \pi v / k}{\pi v / k}\right)^{n \alpha_{k}}=e^{-(1 / 3 !) \pi^{2} \psi v^{2}}, \quad v \neq 0 .
$$

Theorem 4.3. A necessary and sufficient condition for $\left(s_{n, k}^{\left(a_{k}\right)} f\right)$ to converge strongly for any $f \in X_{2 \pi}$ as $n, k \rightarrow \infty$ is that $\lim _{n, k \rightarrow \infty} n \alpha_{k} / k^{2}=\gamma$ exists or equals $\infty$.

If $\gamma \neq 0$ or $\infty$, then for any $\zeta>0$ and $f \in X_{2 \pi}$,

$$
\lim _{n, k \rightarrow \infty}\left\|s_{k}^{\left(\left[\alpha_{k} \zeta\right]\right)} f-\Phi_{\zeta} f\right\|_{X_{2 x}}=0
$$

where the limiting semigroup is given by

$$
\left(\Phi_{\zeta} f\right)(x)=\sum_{\nu} e^{-\zeta \pi^{2} v^{2} y / 6} f_{\nu} e^{i v x}
$$

for $f(x) \sim \sum_{v} \hat{f}_{v} e^{i v x}$

\section{Trigonometric splines} by

Let $n, k$ be positive integers with $n+1 \leqq k, h:=2 \pi / k$, and define a sequence $\left(a_{n, v}\right), v \in \mathbb{Z}$,

$$
a_{n, v}:=\frac{1}{2 \pi i} \prod_{j=0}^{n}\left(\frac{1-\exp i(j-v) h}{v-j}\right), \quad v \in \mathbb{Z},
$$

where the factor whose denominator equals zero is taken to be ih. The terms of the 
sequence $c_{n, v}=0$ if and only if $v=k p+j, j=0,1, \ldots, n, p \in \mathbb{Z}, p \neq 0$. It is known (see Schoenberg [17]), that

$$
M_{n}\left(e^{i v}\right):=\sum_{v} a_{n, v} e^{i v x}, \quad x \in[0,2 \pi]
$$

is a piecewise polynomial function in $e^{i x}$ of degree $n$, with knots at $j h, j=0,1, \ldots, k-1$, which possesses continuous derivatives up to order $n-1$, and is supported on $[0,(n+1) h]$.

A straightforward computation shows that

$$
a_{n, v}=i^{n} e^{i(n+1)((1 / 2) n-v) / 2} d_{v}
$$

where

$$
d_{v} \equiv d_{n, v}:=\frac{2^{n}}{\pi} \prod_{j=0}^{n} \frac{\sin (v-j) h / 2}{(v-j)}, \quad 0 \leqq v \leqq n
$$

the factor whose denominator equals zero is taken to be $h / 2$. Hence

$$
M_{n}\left(e^{i x}\right)=i^{n} e^{i n x / 2} \sum_{v} d_{v} e^{i(v-n / 2)(x-(n+1) h / 2)}
$$

Since $d_{v}=d_{n-v}, v \in \mathbb{Z}$, the function

$$
P_{n}(x):=\sum_{v} d_{n, v} e^{i(v-n / 2)(x-(n+1) h / 2)}, \quad x \in[0,2 \pi),
$$

is a real function supported on the interval $[0,(n+1) h]$ and its restriction to each subinterval $(j h,(j+1) h)$ lies in the linear span of $\left(\sin \frac{1}{2} x\right)^{v}\left(\cos \frac{1}{2} x\right)^{n-v}, v=0,1, \ldots, n$. Clearly

$$
P_{n}(x)=(-i)^{n} e^{-i n x / 2} M_{n}\left(e^{i x}\right), \quad x \in[0,2 \pi),
$$

and we define $P_{n}(x), x \in \mathbb{R}$, by requiring it to be $2 \pi$-periodic. The function $P_{n}$ is called a trigonometric $B$-spline degree $n$ (see $[6,16]$ ). They satisfy the recurrence relation

$$
n P_{n}(x)=2 \sin \frac{1}{2} x P_{n-1}(x)+2 \sin \frac{1}{2}((n+1) h-x) P_{n-1}(x-h) .
$$

Since $P_{0}(x) \geqq 0$, it follows from (5.6) that $P_{n}(x) \geqq 0$.

We are interested in the case $n=2 m$ is an even integer, $m=1,2, \ldots$, where we define

$$
\tau(x) \equiv \tau_{m, k}(x):=P_{2 m}(x+(n+1) h / 2) / d_{m}, \quad x \in \mathbb{R} .
$$

Then

$$
\tau(x)=\sum_{v} \hat{\tau}_{v} e^{i v x}, \quad x \in \mathbb{R},
$$


where

$$
\begin{aligned}
\hat{\tau}_{v} \equiv & \hat{\tau}_{m, k, v}:=d_{v+m} / d_{m} \\
& = \begin{cases}\frac{(m !)^{2}(\sin (m-v) h / 2 \ldots \sin h / 2)(\sin (m+v) h / 2 \ldots \sin h / 2)}{(m-v) !(m+v) !(\sin h / 2 \ldots \sin m h / 2)^{2}}, & |v| \leqq m \\
\frac{k(m !)^{2} \sin (|v|-m) h / 2 \sin (|v|-m+1) h / 2 \ldots \sin (|v|+m) h / 2}{\pi(|v|-m) \ldots(|v|+m)(\sin h / 2 \ldots \sin m h / 2)^{2}}, & |v|>m .\end{cases}
\end{aligned}
$$

The Fourier coefficients $\hat{\tau}_{v}=0$ if and only if $|v|=p k-m, p k-m+1, \ldots, p k+m, p=$ $1,2, \ldots$. In particular, if $k=2 m+1$, then $\hat{\tau}_{v}=0$ for $|\nu| \geqq m+1$, and

$$
\hat{\tau}_{v}=\frac{(m !)^{2}}{(m-v) !(m+v) !}, \quad|v| \leqq m
$$

Therefore

$$
\tau(x)=\sum_{v=-m}^{m} \frac{(m !)^{2}}{(m-v) !(m+v) !} e^{i v x}:=\chi_{m}(x)
$$

are the de la Vallée Poussin kernels and

$$
\left(V_{m} f\right)(x):=\frac{1}{2 \pi} \int_{-\pi}^{\pi} \chi_{m}(x-t) f(t) d t, \quad x \in \mathbb{R}
$$

the de la Vallée Poussin means for a $2 \pi$-periodic integrable function $f$ (see $[1,3,14]$ ). An extension of $(5.11)$ to convolution operators with trigonometric $B$-spline kernels was studied in [7].

Let $\mathscr{T}_{m, k}:=\left\{s \in C^{2 m-1}(\mathbb{R}):\left.s\right|_{((j-1 / 2) h,(j+1 / 2) h)}\right.$ equals a trigonometric polynomial of degree $m\}$. The following results follow from (5.5), (5.6) and the corresponding properties of $M_{n}\left(e^{i x}\right)$ (see [17]).

Proposition 5.1. The function $\tau_{m, k} \in \mathscr{T}_{m, k}$ is even, $2 \pi$-periodic and $\operatorname{supp} \tau_{m, k}=$ $\left[-m-\frac{1}{2} h, m+\frac{1}{2} h\right]$.

Proposition 5.2. The space $\mathscr{T}_{m, k}$ is a linear space of dimension $k$ spanned by $\tau(\cdot-j h), j=0,1, \ldots, k-1$.

Proposition 5.3. For $\alpha=1,2,3, \ldots$, and $j \in \mathbb{Z}$,

$$
\sum_{p} \hat{\tau}_{j+k p}^{a+1} \neq 0
$$

Furthermore, for $|j| \leqq m$, 


$$
\sum_{p} \hat{\tau}_{j+k p}^{\alpha+1}=\hat{\tau}_{j}^{\alpha+1}
$$

Proof. The relation (5.13) follows from (5.9). Hence (5.12) holds for $|j| \leqq m$. For $|j|>m$, the result follows by a similar argument as Schoenberg $([17$, p. 412]).

For $\alpha=0,1, \ldots$, and $f \in X_{2 \pi}\left(f \in C_{2 \pi}\right.$ if $\left.\alpha=0\right)$, let

$$
\left(t_{m, k}^{(\alpha)} f\right)(x):=\frac{1}{k} \sum_{j=0}^{k-1}\left(T_{m, k}^{(\alpha)} f\right)(j h) \tau_{m, k}(x-j h)
$$

where $T_{m, k}^{(\alpha)} f$ is defined by (1.3) with $\phi_{k}=\tau_{m, k}$. By Theorem 2.1 and Proposition 5.3, the restriction $\left.t_{m, k}^{(\alpha)}\right|_{\mathscr{S}_{m, k}} \rightarrow \mathscr{T}_{m, k}$ is bijective. It follows from (2.14) and (2.8) that the nonzero eigenvalues of $t_{m, k}^{(\alpha)}$ and the corresponding eigenfunctions are respectively

$$
\begin{gathered}
\lambda_{m, j}^{(\alpha)}=\lambda_{j}^{(\alpha)}:=\sum_{p} \hat{\tau}_{j+k p}^{\alpha+1}, \\
f_{m, j} \equiv f_{j}:=\frac{1}{k} \sum_{l=0}^{k-1} \omega^{j l} \tau_{m, k}(\cdot-l h), \quad j=0,1, \ldots, k-1 .
\end{gathered}
$$

For convenience, we extend $\lambda_{m, j}^{(\alpha)}$ and $f_{m, j}$ to all $j \in \mathbb{Z}$ by periodicity so that $\lambda_{j+k}=\lambda_{j}$ and $f_{j+k}=f_{j}, j \in \mathbb{Z}$. By (5.13) we have

$$
\lambda_{m, j}^{(\alpha)}=\hat{\tau}_{j}^{a+1} \text { for }|j| \leqq m
$$

Let $E_{m, j}$ be the corresponding normalised eigenfunctions.

Proposition 5.4. The set $\left\{E_{m, j}:-m \leqq j \leqq k-m-1\right\}$ is an orthonormal basis for $\mathscr{T}_{m, k}$. For $|j| \leqq m, E_{m, j}(x)=e^{i j x}$.

Proof. The first part of the assertion follows from Corollary 2.2. The second part follows from Proposition 2.3 since $e^{i j x} \in \mathscr{T}_{m, k}$ and $\hat{\tau}_{m, k, j}>0$ for $|j| \leqq m$.

Remarks. 1. The eigenfunctions $E_{m, j}(x)$ are related to the $r$-flowers of I. J. Schoenberg [17].

2. The operators $T_{m, k}^{(\alpha)}$ and $t_{m, k}^{(\alpha)}$ are related to the de la Vallee Poussin operator $V_{m}$ defined in (5.11). In fact when $k=2 m+1, T_{m, 2 m+1}^{(1)}=V_{m}$ and $T_{m, 2 m+1}^{(a)}$ are products (in the sense of composition) of $V_{m}$. Also, $t_{m, 2 m+1}^{(0)} f$ is a discrete analogue of de la Vallee Poussin means.

It is straightforward to verify that the Fourier coefficients $\hat{\tau}_{m, k, v}$ satisfy (3.2) to (3.6) for $m \geqq 1$. Therefore the results of Theorems 3.4 and 3.5 are applicable to the trigonometric spline operator $t_{m, k}^{\left(a_{k}\right)}$ where the limits in (3.16), (3.17), (3.18) are taken as 
$k \rightarrow \infty$ with $m$ fixed. In fact, the results of Theorem 3.4 also hold for $t_{m, k}^{\left(a_{k}\right)}$ if the limits are taken in such a way that $m, k \rightarrow \infty$ and $m h=2 \pi m / k \rightarrow \theta \in[0, \pi]$. In particular we have

Theorem 5.5. Let $\alpha_{m}, m=1,2,3, \ldots$ be a nondecreasing sequence of positive integers. Then

$$
\lim \left\|t_{m, k}^{\left(\alpha_{m}\right)} f-f\right\|_{X_{2 \pi}}=0 \quad \text { for all } f \in X_{2 \pi}
$$

if and only if

$$
\lim \left(\frac{m \sin (m+1) h / 2}{(m+1) \sin m h / 2}\right)^{\alpha_{m}}=1
$$

where the limit is taken as $m, k \rightarrow \infty$ and $m h \rightarrow \theta \in[0, \pi]$.

Furthermore (5.19) holds if and only if $\alpha_{m}=0(m)$ as $m \rightarrow \infty$.

Proof. The first part of the theorem follows by the same argument as in the proof of (3.17) in Theorem 3.4, with $\hat{\phi}_{k, 1}$ given by

$$
\hat{\tau}_{m, k, 1}:=\frac{m \sin (m+1) h / 2}{(m+1) \sin m h / 2}
$$

Further, a straightforward computation gives

$$
(m+1)\left(1-\hat{\tau}_{m, k, 1}\right)=1+2 m \sin ^{2} \frac{1}{4} h-m \cot \frac{1}{2} m h \sin \frac{h}{2} .
$$

Hence

$$
\hat{\tau}_{m, k, 1}=1-\frac{1}{m+1}\left(1-\frac{m h}{2} \cot \frac{m h}{2}\right)+0\left(h^{2}\right)
$$

Since

$$
1-\frac{m h}{2} \cot \frac{m h}{2} \rightarrow 1-\frac{\theta}{2} \cot \frac{\theta}{2} \neq 0 \text { for } \theta \in[0, \pi]
$$

(5.19) holds if and only if $\alpha_{m}=0(m)$ by (5.21).

\section{REFERENCES}

1. H. BERENS, Interpolationsmethoden zur Behandlung von Approximationsprozessen auf Banachräumen (Lecture Notes in Math. 64, Springer 1968).

2. P. L. Butzer and H. Berens, Semi-Groups of Operators and Approximation (Springer-Verlag 1967). 
3. P. L. Butzer and R. J. Nessel, Fourier Analysis and Approximation, Vol. $l$ (Academic Press 1971).

4. P. J. Davis, Circulant Matrices (Wiley 1979).

5. Franz-Jürgen Delvos, Periodic interpolation on uniform meshes, J. Approx. Theory 51 (1987), 71-80.

6. T. N. T. Goodman and S. L. Lee, $B$-splines on the circle and trigonometric $B$-splines, Proc. Conf. on Approx. Theory and spline functions (S. P. Singh, J. W. H. Bury and B. Watson, eds., St. Johns, Newfoundland, Reidel Pub. Co., 1983), 297-325.

7. T. N. T. Goodman and S. L. Lee, Convolution operators with trigonometric spline kernels, Proc. Edinburgh Math. Soc. 31 (1988), 285-299.

8. Masaru Kamada, Kazuo Toraichi and Ryoich Mori, Periodic spline orthonormal bases, $J$. Approx. Theory 55 (1988), 27-34.

9. S. KARLIN and Z. ZeIgLer, Iteration of positive approximation operators, J. Approx. Theory 3 (1970), 310-399.

10. P. P. Korovkin, Linear Operators and Approximation Theory (Hindustan Publ. 1960).

11. F. LoCHER, Interpolation on uniform meshes by translates of one function and related attenuation factors, Math. Comp. 37 (1981), 403-416.

12. M. J. MARSDEN, An identity for spline functions with applications to variation diminishing spline approximation, J. Approx. Theory 3 (1970), 7-49.

13. G. Meinardus, Periodische splinefunktionen, in Spline functions, Karlsruhe 1975 (K. Böhner, G. Meinardus and W. Schempp, eds., Lecture Notes in Math. 501, Springer 1976).

14. G. Polya and I. J. Schoenberg, Remarks on de la Vallée Poussin means and convex conformal maps on the circle, Pacific J. Math. 8 (1958), 295-334.

15. I. J. Schoenberg, On interpolation by spline functions and its minimal properties, in $O n$ Approximation Theory (Intern. Ser. Numerical Math (ISNM), Birkhauser 5, 1964), 109-129.

16. I. J. Schoengerg, On trigonometric spline interpolation, J. Math. Mech. 13 (1964), 795-825.

17. I. J. Schoenberg, On polynomial splines on the circle $\mathrm{I}$, in Proc. Conf. on Constructive Theory of Functions (Budapest 1972), 403-433.

Department of Mathematics

National University of Singapore

10 Kent Ridge Crescent

SiNGAPORE 0511 